\title{
Gatilhos para transfusão sanguínea
}

\section{Triggers for blood transfusion}

\author{
Mariana Menezes Bochio할 Vanessa Andrea Pincelli ${ }^{1}$; \\ Felipe Ribeiro Botelho Santos ${ }^{2}$; Igor Ferreira ${ }^{3}$; Patrícia Mendes Pereira ${ }^{4 *}$
}

\section{Resumo}

A anemia é uma importante afecção encontrada na medicina e na clínica de animais de companhia, principalmente nos pacientes críticos. As alterações clínicas da anemia geralmente estão relacionadas à menor oxigenação dos tecidos ou aos mecanismos compensatórios a ela associados. A transfusão sanguínea tem o objetivo de impedir os danos de hipóxia causados pela anemia em pacientes criticamente enfermos. Porém, uma vez que a transfusão está sujeita a diversos riscos, ela só deve ser realizada quando realmente necessária. Diversos fatores influenciam a decisão para realização da transfusão sanguínea. O objetivo deste trabalho foi fornecer uma revisão dos principais estudos realizados sobre possíveis gatilhos transfusionais. Entre os exames laboratoriais, o valor de hemoglobina $(\mathrm{Hb})$ é o principal gatilho, variando de acordo com idade do paciente e doenças concomitantes. Além disso, o eletrocardiograma e a dosagem sérica de lactato, associados à $\mathrm{Hb}$, podem ajudar na decisão de quando transfundir.

Palavras-chave: Anemia, hemoglobina, lactato, eletrocardiograma, cães

\begin{abstract}
Anemia is an important condition that is found in the internal medicine, either in humans and animals, especially in critically ill patients. The clinical signs of anemia are usually related to a poor tissue oxygenation or the compensatory mechanisms associated with it. Blood transfusion aims to prevent hypoxic damage in critically ill patients. However, once the blood transfusion is associated to some risks, it should just be done if really necessary. Several factors influence blood transfusion decision. The aim of this study was to review the major studies about transfusion triggers. Among laboratory tests, the hemoglobin $(\mathrm{Hb})$ is the main trigger but it depends on patient age and concomitant disease. In addition, electrocardiogram (ECG) and serum lactate with $\mathrm{Hb}$ can help to decide when to transfuse.
\end{abstract}

Key words: Anemia, hemoglobin, lactate, electrocardiogram, dogs

\footnotetext{
${ }^{1}$ Médicas Veterinárias M.e. em Ciência Animal, Universidade Estadual de Londrina, UEL, Londrina, PR. E-mail: marianabochio@, hotmail.com; vapincelli@hotmail.com

${ }^{2}$ Médico Veterinário Residente de Anestesiologia, Universidade Federal de Santa Maria, UFSM, Santa Maria, RS. E-mail: feliperbsantos@hotmail.com

${ }^{3}$ Médico Veterinário Residente de Clínica Médica de Animais de Companhia, UEL, Londrina, PR. E-mail: igorvetuel@hotmail. com

${ }^{4}$ Médica Veterinária Prof ${ }^{\mathrm{a}} \mathrm{Dr}^{\mathrm{a}}$ da área de Clínica Médica de Animais de Companhia, Dept ${ }^{\mathrm{o}}$ de Clínicas Veterinárias, UEL, Londrina, PR. E-mail: pmendes@uel.br

* Autor para correspondência
} 


\section{Anemia}

$\mathrm{O} \mathrm{O}_{2}$ é transportado pelo sangue de duas formas: por meio da hemoglobina $(\mathrm{Hb})$ nos eritrócitos e cerca de $3 \%$ dissolvido no plasma. A real quantidade de oxigênio no sangue arterial $\left(\mathrm{CaO}_{2}\right)$ é determinada pela concentração de hemoglobina no sangue, pela saturação de oxigênio na $\mathrm{Hb}\left(\mathrm{SaO}_{2}\right)$, pela capacidade da $\mathrm{Hb}$ de carrear o oxigênio, pela pressão parcial de oxigênio arterial $\left(\mathrm{PaO}_{2}\right)$ e pela solubilidade de oxigênio no plasma (SHANDER, 2004).

Anemia é a incapacidade do sangue de suprir aos tecidos oxigenação adequada para tornar viável a função metabólica e se caracteriza pela diminuição da contagem de eritrócitos, do volume globular (VG) e da concentração de hemoglobina $(\mathrm{Hb})$ abaixo dos valores de referência para uma determinada espécie. Esta alteração resultará na diminuição da oxigenação dos tecidos orgânicos e é considerada uma manifestação clínica que pode ser causada por destruição (hemólise), perda (hemorragia), diminuição da produção de eritrócitos, ou combinações desses eventos (AIRD, 2000).

Para minimizar os efeitos da hipóxia tecidual causada pela anemia, ocorre a ativação de mecanismos compensatórios, como a vasoconstrição periférica, o aumento do retorno venoso e do débito cardíaco, a redistribuição do fluxo sanguíneo para órgãos vitais e o aumento da concentração de 2,3-difosfoglicerato (2,3-DPG) nas hemácias, que diminui a afinidade do oxigênio pela hemoglobina facilitando a sua liberação para os tecidos (HÉBERT et al., 2004).

As alterações clínicas em pacientes anêmicos geralmente estão relacionadas a esses mecanismos compensatórios e à menor oxigenação dos tecidos. Os principais sinais clínicos observados são a palidez de mucosas, apatia, menor tolerância ao exercício, aumento da frequência respiratória e frequência cardíaca e a presença de sopros induzidos pela maior turbulência do sangue. A gravidade dos sinais clínicos geralmente está associada ao tempo e severidade da instalação do quadro anêmico (AIRD,
2000; HURCOMBE; MUDGE; HINCHCLIFF, 2007).

A anemia deve ser classificada pela sua intensidade, tempo de aparecimento e de acordo com a resposta medular (regenerativa ou não regenerativa). Segundo a gravidade, em cães, a anemia é classificada em leve (VG 30 -37\%), moderada (VG 20-29\%), severa (VG 13-19\%) e muito severa ( $\mathrm{VG}<13 \%)$. Já em gatos, a anemia é considerada leve entre 20 e $26 \%$ de VG, moderada entre 14 e $19 \%$, severa entre 8 e $13 \%$ e muito severa para valores abaixo de 8\% (TVEDTEN; WEISS, 2000).

Quanto ao tempo de aparecimento, a anemia é classificada em aguda ou crônica. Em animais com anemia crônica por insuficiência renal ou disfunção de medula óssea ocorre, através de mecanismos compensatórios, certo grau de compensação da hipoxêmia. Nos animais com perda de sangue ou hemólise aguda, os sinais clínicos são mais acentuados, pois não há tempo suficiente para uma compensação mais adequada do organismo (WEISS; WARDROP, 2010).

A resposta da medula óssea frente ao quadro de anemia é fundamental para a abordagem diagnóstica e para determinar o prognóstico do paciente. Ela classifica a anemia em regenerativa ou não regenerativa. Esta classificação é feita principalmente através da contagem de reticulócitos no esfregaço sanguíneo periférico e pela avaliação citológica da medula óssea. Animais com anemia regenerativa têm produção adequada na medula óssea, portanto, as causas de anemia que devem ser investigadas são a perda de sangue ou a hemólise (TVEDTEN; WEISS, 2000).

Em animais com anemia arregenerativa é preciso avaliar a medula óssea por citologia para verificar a produção de eritrócitos e pesquisar possíveis etiologias. As causas de anemia arregenerativa geralmente são doenças crônicas, como doenças inflamatórias, doença renal, hepatopatias, neoplasias, perda de sangue crônica, deficiências 
nutricionais, intoxicações medicamentosas ou químicas, hipoplasia ou aplasia da medula óssea (FERNANDEZ; GRINDEN, 2000).

\section{Transfusão Sanguínea}

Atualmente, estudos mostram que cerca de 95\% dos pacientes admitidos em Unidades de Terapia Intensiva (UTI's), têm níveis de hemoglobina abaixo do normal (FERREIRA; FERREIRA; PELANDRÉ, 2005). Destes pacientes, 37 a $44 \%$ recebem uma ou mais transfusões sanguíneas (SIGRIST, 2008).

A transfusão sanguínea impede os danos causados pela hipóxia tecidual anêmica no paciente crítico. O seu objetivo é aumentar a sobrevida do paciente enquanto se procura o diagnóstico e a terapia se torne efetiva ou também aumentar a qualidade de vida dos pacientes com doenças crônicas irreversíveis (PEREIRA, 2007).

Morikawa et al. (2010), avaliaram 77 transfusões em cães, sendo 52 de sangue total e 25 de concentrado de hemácias, e constataram que ambos componentes promoveram melhora dos parâmetros clínicos que foram avaliados e monitorados durante toda transfusão (temperatura, frequência cardíaca e respiratória, coloração de mucosas e tempo de preenchimento capilar). Além disso, a transfusão proporcionou melhora clínica aparente no animal a partir de duas horas do início do procedimento.

Embora muitas vezes a transfusão sanguínea seja necessária ao paciente, é importante lembrar que é um procedimento sujeito a muitos riscos (GROVER et al., 2006; PEREIRA, 2007). Segundo Pincelli et al. (2010), a incidência de reações transfusionais agudas em cães varia de 2,9 a 28,49\% na literatura. Em seu estudo, que avaliou 113 transfusões sanguíneas em cães (71 de sangue total, 26 de concentrado de hemácias e 16 de plasma fresco congelado) foi encontrado uma incidência de 13,27\% de reações transfusionais agudas representadas por: reação urticariforme $(9 / 15)$, reação hemolítica aguda (2/15), sobrecarga circulatória (2/15), hipertermia (1/15) e vômito (1/15).
No homem, menos de $3 \%$ das transfusões sanguíneas provocam reação transfusional. As mais comuns são a reação não hemolítica febril ou hipertermia, a reação alérgica, a contaminação bacteriana e a lesão pulmonar aguda (TRALI), conforme revisado por Pincelli et al. (2010). A TRALI é caracterizada por um edema pulmonar agudo não cardiogênico que pode ocorrer após a transfusão de hemácia, levando a uma grave dispneia e hipoxemia e eventualmente pode ocorrer febre, hipotensão e cianose (PRITTIE, 2010). É a principal causa de mortalidade em seres humanos relacionada à transfusão (TOCCI, 2010), em animais, a TRALI foi relatada apenas em modelos experimentais (PRITTIE, 2010).

Além disso, a transfusão é responsável por alterações complexas do sistema imune, produzindo imunomodulações, transformando-se em um fator de risco para infecção hospitalar ou recidiva de neoplasias (GOMES, 2006). O mecanismo de imunomodulação após a transfusão não está ainda totalmente elucidado e provavelmente seja multifatorial. Sabe-se que após a transfusão sanguínea ocorre a diminuição na função das células natural killer e dos macrófagos, induzindo a supressão dos linfócitos T (PRITTIE, 2010).

Portanto, a transfusão sanguínea nunca deve ser feita de forma desnecessária tanto em pacientes humanos, quanto veterinários (GROVER et al., 2006; PEREIRA, 2007).

Existe atualmente um debate sobre os principais critérios para a indicação de uma transfusão sanguínea tanto em humanos como em animais, uma vez que, dependendo da causa da anemia e da quantidade e rapidez do sangue perdido, a anemia pode ser bem tolerada (PRITTIE, 2010).

\section{Gatilhos Transfusionais}

\section{Hemoglobina em humanos}

A Hb é o principal fator envolvido na capacidade de transporte de oxigênio e, portanto, é um 
importante gatilho para a decisão de uma transfusão sanguínea (SIGRIST, 2008).

Historicamente, acreditava-se que a concentração de hemoglobina deveria ser mantida em níveis superiores a $10,0 \mathrm{~g} / \mathrm{dL}$ para garantir bom aporte de oxigênio aos tecidos. Atualmente em humanos, muitos autores têm recomendado transfusão de concentrado de hemácias $(\mathrm{CH})$ em pacientes criticamente enfermos, com concentração de hemoglobina inferior a 7,0 g/dL, evitando transfusões com valores de hemoglobina superiores a 10,0 g/dL (MCFARLAND, 1999; VINCENT; YALAVATTI, 2003; NAPOLITANO; CORWIN, 2004; FERREIRA; FERREIRA; PELANDRÉ, 2005; SIGRIST, 2008; PRITTIE, 2010; WANG; KLEIN, 2010, SAKR et al., 2010).

Hébert et al. (1999), avaliaram um total de 838 pacientes críticos admitidos na unidade de terapia intensiva com hemoglobina inicial de $9 \mathrm{~g} / \mathrm{dL}$. Os pacientes foram aleatoriamente divididos em dois grupos: um grupo restritivo (gatilho transfusional de $7 \mathrm{~g} / \mathrm{dL}$ ) e um grupo liberal (gatilho transfusional de $10 \mathrm{~g} / \mathrm{dL}$ ). A mortalidade em 30 dias após a transfusão foi menor no grupo restritivo, porém a diferença não foi significativa entre os grupos. Entretanto, quando os pesquisadores analisaram subgrupos incluindo pacientes menos gravemente enfermos (APACHE II $\leq 20)$ ou mais jovens $(<55$ anos), demonstraram uma redução significativa da mortalidade nos pacientes que pertenciam ao grupo restritivo. Entre os pacientes com doença cardíaca a mortalidade foi muito próxima entre os dois grupos.

Segundo a PRACTICE guidelines for perioperative blood transfusion and adjuvant therapies (2006), é recomendada no período operatório a transfusão sanguínea para níveis de hemoglobina menores do que $6 \mathrm{~g} / \mathrm{dL}$ em pacientes jovens e saudáveis, evitando assim as lesões por hipoxemia. Além disso, estudos comprovam que a mortalidade nos pacientes cirúrgicos aumenta significativamente quando os níveis de hemoglobina decaem para menos que $5 \mathrm{~g} / \mathrm{dL}$ (MCFARLAND, 1999; SIGRIST, 2008).
Mathru et al. (2006), avaliaram 19 pacientes submetidos a cirurgias eletivas e demonstraram que uma redução dos níveis de hemoglobina para 5,9 g/ dL mantendo a volemia, reduz a oferta e consumo de oxigênio esplâncnico e pré-portal. Essa redução foi acompanhada pelo aumento dos níveis séricos de lactato e aumento da taxa de b-hidroxibutirato/ acetato. Estes achados sugerem que o risco de hipóxia do trato gastrointestinal pode estar sendo subestimado durante manutenção desses baixos níveis de hemoglobina.

Em um estudo retrospectivo realizado por Carson et al. (2002), foi avaliada a mortalidade e a morbidade após a cirurgia em pacientes com baixas concentrações de hemoglobina que recusaram receber a transfusão sanguínea por motivos religiosos. Neste estudo eles encontraram mortalidades de $100 \%$ ( $\mathrm{Hb}$ entre 1,1 e $2,0 \mathrm{~g} / \mathrm{dL}$ ), $54,2 \%$ (Hb entre 2,1 e $3,0 \mathrm{~g} / \mathrm{dL}$ ), $25 \%$ (Hb entre 3,1 e 4,0g/dL), 34,4\% (Hb entre 4, 1 e 5,0g/dL), 9,3\% ( $\mathrm{Hb}$ entre 5,1 e $6,0 \mathrm{~g} / \mathrm{dL}), 8,9 \%$ ( $\mathrm{Hb}$ entre 6,1 e $7,0 \mathrm{~g} / \mathrm{dL}$ ) e nenhuma mortalidade ocorreu em pacientes com $\mathrm{Hb}$ de 7,1 a 8,0g/dL. Neste estudo também concluíram que a diminuição de cada $1 \mathrm{~g} / \mathrm{dL}$ na concentração de hemoglobina faz com que o risco de morte aumente em duas vezes e meia. Além disso, encontraram altas taxas de morbidades nos pacientes anêmicos, como infarto de miocárdio, insuficiência cardíaca congestiva e infecções.

\section{Hemoglobina em animais}

No cão e no gato os parâmetros de concentração de $\mathrm{Hb}$ para transfusão sanguínea ainda permanecem incertos (SIGRIST, 2008). Assim como em humanos, parâmetros clínicos são mais importantes do que resultados laboratoriais na decisão (PEREIRA; RAMALHO, 2001; SIGRIST, 2008). Uma forma de estudar experimentalmente a anemia e chegar a um valor de hemoglobina crítica, ou seja, um valor abaixo do qual a hipóxia tecidual não é tolerada, é por meio da diluição intencional do sangue (hemodiluição), uma técnica na qual uma 
quantidade de sangue é removida do indivíduo, porém o volume é mantido através da infusão simultânea de soluções cristalóides ou coloides e posteriormente o sangue pode ser reinfundido (GEHA; BAUE, 1978; JAMNICKI et al., 2003; SHANDER, 2004; CHAMPION, 2008).

Sob as condições de uma anemia aguda normovolêmica (hemodiluição), o consumo de O2 pelos tecidos (VO2) é mantido pelo aumento do débito cardíaco e pelo aumento da liberação de oxigênio para os tecidos (aumento da síntese 2,3-DPG). Os valores de VG e $\mathrm{Hb}$ chamados de críticos são encontrados quando estes mecanismos compensatórios não são suficientes em manter a oxigenação adequada dos tecidos (SHANDER, 2004). Somente em valores muito baixos de $\mathrm{Hb}$, com grande diminuição de aporte de oxigênio (DO2), ocorre a falha desses mecanismos, diminuição do VO2 e conseqüente aumento na produção de lactato sérico, indicando uma insuficiência na oxigenação dos tecidos devido à grave anemia normovolêmica (JAMNICKI et al., 2003).

Segundo Jamnicki et al. (2003), o DO2 é considerado crítico quando ocorrem fluxos de oxigênio entre 4 a $10 \mathrm{~mL} / \mathrm{kg} / \mathrm{min}$, em cães anestesiados. Este valor corresponde a valores de $\mathrm{Hb}$ entre 2,5 a $4,1 \mathrm{~g} / \mathrm{dL}$ ou 18 a $26 \%$ do valor basal $\mathrm{de} \mathrm{Hb}$. Entretanto, é importante salientar que o DO2 crítico varia de acordo com o estado de consciência, por exemplo, em pacientes não anestesiados, o VO2 se torna maior, devido ao aumento do metabolismo (WANG; KLEIN, 2010).

Em babuínos anestesiados submetidos à hemodiluição aguda, a função cardiovascular foi mantida até a concentração de $\mathrm{Hb}$ alcançar valores entre 3 e $5 \mathrm{~g} / \mathrm{dL}$, detectando-se então, alterações por hipóxia no exame de eletrocardiograma, como alterações no seguimento ST (WILKERSON et al., 1988). Entretanto, 12 cães submetidos a esse procedimento com estenose coronária experimental desenvolveram alterações isquêmicas em concentrações de $\mathrm{Hb}$ de 7 a $10 \mathrm{~g} / \mathrm{dL}$, evidenciando que pacientes com cardiopatias são mais sensíveis a anemia (GEHA; BAUE, 1978).

Schwartz, Franstz e Shoemaker (1981), utilizando a hemodiluição em um modelo canino, encontraram o valor crítico de VG de 10\%. Já Hoeft, Wietasch e Sonntag (1995) avaliaram a correlação entre o VG, o fluxo coronariano e a oxigenação do miocárdio e verificaram que quando o consumo de oxigênio era normal, o VG crítico foi de $14 \%$ e $\mathrm{Hb}$ de $4,7 \mathrm{~g} / \mathrm{dL}$, porém quando a necessidade de consumo oxigênio estava aumentada, como em pacientes em recuperação pós-operatória, o VG crítico era de $21 \%$ ( $\mathrm{Hb}$ de $7 \mathrm{~g} / \mathrm{dL})$.

Além das cardiopatias, outras afecções também podem fazer com que o paciente seja particularmente mais vulnerável a hipóxia anêmica. Alguns estudos foram realizados para descobrir o grau de tolerância a anemia em pacientes com doenças cerebrovasculares, doença pulmonar obstrutiva crônica e insuficiência renal. Entretanto, ainda não foram encontrados parâmetros universais para realização da transfusão sanguínea nesses pacientes (PRITTIE, 2010).

Vale ressaltar que os valores críticos de $\mathrm{Hb}$ irão variar com diversos fatores individuais, tais como idade, severidade da doença e doenças préexistentes (VINCENT; YALAVATTI, 2003). O nível de hemoglobina como parâmetro isolado não deve ser utilizado na indicação da transfusão sanguínea e sugere-se que a transfusão seja subordinada a uma avaliação clínica baseada em parâmetros como sinais vitais, condições hemodinâmicas e presença de sangramento ou hemólise (PEREIRA; RAMALHO, 2001; FERREIRA; FERREIRA; PELANDRÉ, 2005).

\section{Gatilho em hemorragias}

Em humanos, trabalhos clínicos experimentais têm mostrado que perdas de até $75 \%$ de massa eritrocitária podem ser toleradas, desde que seja mantida a volemia (FAGGIONI et al., 1999). 
Hemorragias com perdas de cerca de $30 \%$ do volume sanguíneo são freqüentemente fatais, tanto em humanos (FAGGIONI et al., 1999), como em cães (cerca de $30 \mathrm{~mL} / \mathrm{kg}$ ) e gatos (cerca de 20 $\mathrm{mL} / \mathrm{kg}$ ) (KRISTENSEN; FELDMAN, 1995). Nas perdas menores que $30 \%$, a reposição com soluções cristalóides devem ser utilizadas para reexpandir o volume. Porém, em perdas maiores, além de cristalóides, os produtos sanguíneos devem obrigatoriamente ser utilizados (FAGGIONI et al., 1999).

Contudo, a perda sanguínea é difícil de ser estimada, podendo ser feita por meio da freqüência cardíaca, pulso, frequência respiratória, débito urinário e pelo estado de consciência do paciente (FAGGIONI et al., 1999).

\section{O uso de Exames Complementares no Gatilho Transfusional}

\section{Lactato}

Nas condições de hipóxia, o piruvato é preferencialmente reduzido a lactato e a relação entre lactato e piruvato se eleva. O lactato é produzido em todos os tecidos, entretanto, os músculos, o cérebro, as hemácias e a medula renal são responsáveis pela maior produção no organismo (CICHOTA, 2007).

O lactato sérico pode proporcionar a avaliação do grau de oxigenação dos tecidos, uma vez que a produção de lactato é inversamente proporcional à oxigenação tecidual, sendo o resultado do catabolismo anaeróbico da glicose (RABELO; CROWE; 2005; ALLEN; HOLM, 2008). Ou seja, a oxigenação reduzida pelos baixos níveis de hemoglobina resulta em hipóxia, a qual aciona o metabolismo anaeróbico, caracterizando uma correlação inversa entre os níveis de lactato e hemoglobina (CICHOTA, 2007).

Em humanos, os níveis normais de lactato no sangue arterial são de aproximadamente 0,62 $\mathrm{mmol} / \mathrm{L}$, enquanto que no sangue venoso os níveis são ligeiramente mais elevados, da ordem de 0,99
mmol/L (CICHOTA, 2007).

Nos animais, Lagutchik et al. (1996) avaliaram a concentração de lactato no sangue venoso de cães sadios e de cães acometidos por doenças agudas e demonstraram que a concentração média de lactato nos cães clinicamente sadios era de $1,38 \mathrm{mmol} / \mathrm{L}$, enquanto que nos cães doentes que sobreviveram foi de $2,48 \mathrm{mmol} / \mathrm{L}$ e nos doentes que vieram à óbito foi de $3,85 \mathrm{mmol} / \mathrm{L}$.

Embora o lactato seja um indicador de hipóxia tecidual e sua dosagem possa ser utilizada como valor prognóstico, indicando as chances de sobrevida nos casos em que há uma diminuição desse substrato (KOLISKI et al., 2005; NEL et al., 2004; RABELO; ARNOLD; ALSUA, 2009), não existem muitos estudos do lactato como um gatilho transfusional.

Holahan, BrowneDrobatz(2010), comoobjetivode verificarem a associação do lactato com o prognóstico e a resposta a transfusão sanguínea, avaliaram 173 cães com anemia hemolítica imunomediada, dosando o lactato desses animais na admissão, seis, 12 e 24 horas após. A mediana dos valores de lactato na admissão dos cães que sobreviveram foi de $2,9 \mathrm{mmol} / \mathrm{L}$, significativamente menor em relação aos cães que vieram a óbito $(5,1 \mathrm{mmol} / \mathrm{L})$ e dos cães que foram eutanaziados (4,7 mmol/L). Além disso, a mediana da concentração de lactato em todos os outros diferentes tempos avaliados foi significativamente menor nos sobreviventes. Os cães que receberam transfusão de concentrado de hemácias durante as 6 horas após a admissão obtiveram uma maior e significativa mudança na dosagem de lactato $(1,9 \mathrm{mmol} / \mathrm{L} ; 0,8$ a $2,6)$ em relação àqueles cães que não realizaram esse procedimento (1,2 $\mathrm{mmol} / \mathrm{L}$; 0,3 a 2,0 $\mathrm{mmol} / \mathrm{L})$. Para os autores, mensurações seriadas de lactato sérico além de fator prognóstico, são úteis como guia na terapia transfusional.

\section{Eletrocardiograma}

O eletrocardiograma (ECG) avalia a frequência e o ritmo cardíaco, além da duração e amplitude 
das ondas P-QRS-T e intervalos P-R, S-T e Q-T (TILLEY, 1992; BOARI et al., 2003).

Em quadros anêmicos, as alterações encontradas devido à hipóxia do miocárdio são arritmias ventriculares, alterações no segmento $\mathrm{S}-\mathrm{T}$, aumento da FC, da onda $\mathrm{T}$ e do segmento Q-T (TILLEY, 1992; BOARI et al., 2003). Segundo Mueller et al. (2006), em todos pacientes com anemias severas e dor torácica frequente, devem ser realizadas avaliações eletrocardiográficas periódicas.

Boarietal.(2003), avaliaramum paciente anêmico ( $\mathrm{Hb} 4,7 \mathrm{~g} / \mathrm{dL}$ ) com histórico de infarto do miocárdio há dez anos que apresentava depressão significativa do segmento S-T (infra-desnivelamento) no ECG, após duas transfusões de concentrado de hemácias e elevação da concentração de $\mathrm{Hb}$ para $7,1 \mathrm{~g} / \mathrm{dL}$, o segmento S-T retornou a linha de base no ECG. Para Boari et al. (2003), o ECG não invasivo pode ser um método útil e simples para avaliar a eficácia da transfusão sanguínea em pacientes anêmicos com infarto do miocárdio.

Weiskopf et al. (2003), avaliaram 55 eletrocardiogramas de indivíduos normais que foram submetidos à severa e aguda hemodiluição $(\mathrm{Hb} 5 \mathrm{~g} /$ dl) e observaram infra-desnivelamento do segmento
S-T nos indivíduos com aumento de FC superior a $133 \%$, sugerindo hipóxia reversível do miocárdio.

Em contrapartida, um trabalho realizado em cães submetidos à hemodiluição aguda até níveis de volume globular igual ou inferior a $18 \%$, não foram observados quaisquer alterações eletrocardiográficas que evidenciassem a hipóxia do miocárdio (CHAMPION, 2008).

\section{Outros gatilhos}

Para Gomes (2006), os sinais globais de oxigenação inadequada que podem ser incorporados como gatilhos transfusionais fisiológicos em humanos incluem a instabilidade hemodinâmica com hipotensão e taquicardia, a saturação venosa mista de oxigênio (SVO2) menor do que $50 \%$, a pressão parcial venosa mista de oxigênio (PVO2) menor do que $32 \mathrm{mmHg}$, as alterações eletrocardiográficas recentes do segmento ST e anormalidades de parede no ecocardiograma (ECO). Gomes (2006) sugere indicações dos gatilhos transfusionais fisiológicos ou baseados em níveis de hemoglobina, mostrando onde existe base de evidências e estabelecendo possíveis diferenças na avaliação durante o período perioperatório e na unidade de terapia intensiva (Tabela 1).

Tabela 1. Gatilhos transfusionais fisiológicos em humanos e baseados em níveis de hemoglobina.

\begin{tabular}{|c|c|c|}
\hline Gatilho transfusional & Baseado em evidências & Intraoperatório e UTI \\
\hline \multicolumn{3}{|l|}{ Fisiológicos } \\
\hline Hipotensão Relativa & Sim & Sim \\
\hline Taquicardia Relativa & Sim & Sim \\
\hline Recente depressão ST $>1,0 \mathrm{mv}$ & Sim & Sim \\
\hline Recente elevação ST > 2,0 mv & Sim & Sim \\
\hline Recente anormalidade no ECO & Sim & Sim \\
\hline $\mathrm{PVO}_{2}-\mathrm{mmHg}$ & $<25$ & $<32$ \\
\hline $\mathrm{SVO}_{2}-\%$ & $<50$ & $<60$ \\
\hline \multicolumn{3}{|l|}{ Nível de hemoglobina g/dL } \\
\hline Todos os pacientes & 6 & 7 \\
\hline Pacientes $>80$ anos & $?$ & 8 \\
\hline Doença coronariana ou ICC & $?$ & 8 \\
\hline $\mathrm{SpO} 2<90 \%$ & $?$ & 9 \\
\hline Febre/Hipermetabolismo & $?$ & 8 \\
\hline
\end{tabular}

Fonte: Gomes (2006). 


\section{Conclusão}

Em humanos, embora ainda existam dúvidas de quando realizar uma transfusão sanguínea em cada paciente, os gatilhos transfusionais foram estudados e atualmente a transfusão pode ser indicada para pacientes com $\mathrm{Hb}$ inferior a $7 \mathrm{~g} / \mathrm{dL}$. Em pacientes que tenham a $\mathrm{Hb}$ entre 7 e $9 \mathrm{~g} / \mathrm{dL}$ a transfusão é realizada caso o paciente seja idoso, cardiopata ou tenha graves afecções concomitantes. Muitos autores concordam que ela não deve mais ser realizada em pacientes com $\mathrm{Hb}$ maior que $10 \mathrm{~g} / \mathrm{dL}$, como se pensava anteriormente. Nos animais, os estudos experimentais têm mostrado que eles podem tolerar a anemia até que os níveis de hemoglobina atinjam valores entre 3 e $5 \mathrm{~g} /$ $\mathrm{dL}$, exceto em animais com estenose coronária experimental, que já demonstram alterações por hipóxia com valores entre 7 e 10g/dL. Entretanto, faltam estudos clínicos com esses animais para que se descubram os reais gatilhos transfusionais na medicina veterinária, principalmente nos pacientes gravemente acometidos.

\section{Referências}

AIRD, B. Clinical and hematologic manifestations of anemia. In: FELDMAN, B. F.; ZINKL, J. G.; JAIN, N. C. Schalm's veterinary hematology. 5. ed. Philadelphia: Lippincott Williams \& Wilkins, 2000, p. 140-142.

ALLEN, S. E.; HOLM, J. L. Lactate: physiology and clinical utility. Journal of Veterinary Emergency and Critical Care, San Antonio, v. 18, n. 2, p. 123-132, 2008.

BOARI, B.; CHIERICI, F.; LA CECILIA, O.; PORTALUPPI, F.; MANFREDINI, R. ST segment monitoring as a predictor of reperfusion after blood transfusion in a patient with acute non-Q-wave myocardial infarction. The American Journal of Emergency Medicine, Nova York, v. 21, n. 5, p. 449, 2003.

CARSON, J. L.; NOVECK, H.; BERLIN, J. A.; GOULD, S. A. Mortality and morbidity in patients with very low postoperative $\mathrm{Hb}$ levels who decline blood transfusion. Transfusion Practice, Baltimore, v. 42, n. 7, p. 812- 818, 2002.

CHAMPION, T. Avaliação da função cardíaca em cães com anemia normovolêmica aguda experimental. 2008.
Dissertação (Mestrado na Área de Concentração em Clínica Médica Veterinária) - Universidade Estadual Paulista. Faculdades de Ciências Agrárias e Veterinárias, Jaboticabal.

CICHOTA, L. C. Avaliação da albumina modificada pela isquemia na anemia associada a doença renal crônica. 2007. Dissertação (Mestrado na área de Ciências Farmacêuticas) - Universidade Federal de Santa Maria. Centro de Ciências da Saúde, Santa Maria.

FAGGIONI, L. P. C.; COVAS, D. T.; FILHO, E. D. B.; PÁDUA, M. A.; TOBIAS, R. A.; ROSA, M. J.; PAPA, F. Transfusão maciça: uma abordagem didática para graduandos. Medicina, Ribeirão Preto, v. 32, p. 438-443, 1999.

FERNANDEZ, F. R.; GRINDEN, C. B. Reticulocyte response. In: FELDMAN, B. F.; ZINKL, J. G.; JAIN, N. C. Schaml's veterinary hematology. 5. ed. Filadélfia: Lippincott Willians \& Wilkins, 2000. p. 110-116.

FERREIRA, J. S.; FERREIRA, L. P. V.; PELANDRÉ, G. L. Transfusão de concentrado de hemácias em Unidade de Terapia Intensiva. Revista Brasileira de Hematolologia e Hemoterapia, São José do Rio Preto, v. 27, n. 3, p. 179182,2005

GEHA, A. S.; BAUE, A. E. Graded coronary stenosis and coronary flow during acute normovolemic anemia. World Journal of Surgery, Nova York, v. 2, n. 5, p. 645652, 1978.

GOMES, J. M. A. Anemia no perioperatório- quando e a quem transfundir? In: CAVALCANTI, I. L.; CANTINHO, F. A. F.; NASSAD, A. R. Medicina perioperatória. Rio de Janeiro: SAERJ, 2006. p. 267-279.

GROVER, M.; TALWALKAR, S.; CASBARD, A; BORALESSA, M.; CONTRERAS, M.; BORALESSA, H.; BRETT, S.; GOLDHILL, D. R.; SONI, N. Silent myocardial ischemia and hemoglobin concentration: a randomized controlled trial of transfusion strategy in ower limb arthroplasty. Vox Sanguinis, Basileia, v. 90, p. 105-112, 2006.

HÉBERT, P. C.; VAN DER LINDEN, P.; BIRO, G.; HU, L. Q. Physiologic aspects of anemia. Critical Care Clinics, Philadelphia, v. 20, n. 2, p. 187-212, 2004.

HÉBERT, P. C.; WELLS, G.; BLAJCHMAN, M. A.; MARSHALL, J.; MARTIN, C.; PAGLIARELLO, G.; TWEEDDALE, M.; SCHWEITZER, I.; YETISIR, E. A multicenter, randomized, controlled clinical trial of transfusion requirements in critical care. The New England Journal of Medicine, Massachusetts, v. 340, n. 6, p. 409-417, 1999.

HOEFT, A.; WIETASCH, J.; SONNTAG, H. Theoretical limits of permissive anemia. Zentralblatt für Chirurgie, 
Lípsia, v. 120, n. 8, p. 604-613, 1995.

HOLAHAN, M. L.; BROWN, A. J.; DROBATZ, K. J. The association of blood lactate concentrationwith outcome in dogs with idiopathic immune-mediated hemolytic anemia: 173 cases (2003-2006). Journal of Veterinary Emergency and Critical Care, San Antonio, v. 20, n. 4, p 413-420, 2010.

HURCOMBE, S. D.; MUDGE, M. C.; HINCHCLIFF, K. W. Clinical and clinicopathologic variables in adult horses receiving blood transfusions: 31 cases (19992005). Journal of American Veterinary Medicine Association, Schaumburg, v. 231, n. 2, p. 267-274, 2007.

JAMNICKI, M.; KOCIAN, R.; LINDEN, P. V.; ZAUGG, M.; SPAHN, D. R. Acute normovolemic hemodilution: physiology, limitations, and clinical use. Journal of Cardiothoracic and Vascular Anesthesia, Filadélfia, v. 17, n. 6, p. 747-754, 2003.

KOLISKI, A.; CAT, I.; GIRALDI, D. J.; CAT, M. L. Lactato sérico como marcador prognóstico em crianças gravemente doentes. Jornal de Pediatria, Rio de Janeiro, v. 81, n. 4, p. 287-292, 2005.

KRISTENSEN, A. T.; FELDMAN, B. F. General principles of small animal blood component administration. In: FELDMAN, E. C. Textbook of veterinary internal medicine. 4. ed. Filadélfia: WB Saunders, 1995. p. 1277-1290.

LAGUTCHIK, M. S.; OGILVIE, G. K.;WINGFIELD, W. E.; HACKETT, T. B. Lactate kinetics in veterinary critical care: A review. Journal of Veterinary Emergency and Critical Care, San Antonio, v. 6, n. 2, p. 81-95, 1996.

MATHRU, M.; SALANKI, D. R.; WOODSON, L. C.; FRUNSTON, J. C.; OZKAN, O.; HENKEL, S. N.; Practice guidelines for perioperative blood transfusion and adjuvant therapies. An updated report by the american society of anesthesiologists task force on perioperative blood transfusion and adjuvant therapies. Anesthesiology, Filadélfia, v. 105, n. 1, p. 198-208, 2006.

MCFARLAND, J. G. Perioperative blood transfusions: indications and options. American College of Chest Physicians, Chicago, n. 115, p. 113-121, 1999. Supplement 2.

MORIKAWA, M. K.; BOCHIO, M. M.; PINCELLI, V. A.; FREIRE, R. L.; PREREIRA, P. M. Monitoração e avaliação clínica da eficácia da transfusão de sangue total e concentrado de hemácias em cães. Pesquisa Veterinária Brasileira, Seropédica, v. 30. n. 8, p. 665-669, 2010.

MUELlER, B. U.; MARTIN, K. J.; DREYER, W.; BEZOLD, L. I.; MAHONEY, D. H. Prolonged QT interval in pediatric sickle cell disease. Pediatric Blood Cancer, Hoboken, v. 47, n. 6, p. 831-833, 2006.
NAPOLITANO, M. L.; CORWIN, H. L. Efficacy of red blood cell transfusion in the critically ill. Critical Care Clinics, Philadelphia, v. 20, n. 2, p. 255-268, 2004.

NEL, M.; LOBETTI, R. G.; KELLER, N.; THOMPSON, P. N. Prognostic value of blood lactate, blood glucose, and hematocrit in canine babesiosis. Journal of Veteterinary Internal Medicine, United Kingdom, v. 18, n. 4, p. 471476, 2004.

PEREIRA, P. M. Transfusão em cães e gatos. In: LOPES, S. T. A.; BIONDO, A. W.; SANTOS, A. P. Manual de patologia clínica veterinária. 3. ed. Santa Maria: UFSM, 2007. p. 98-103.

PEREIRA, P. M.; RAMALHO, F. S. Transfusão sanguínea. Revista Clínica Veterinária, São Paulo, n. 34, Ano 6, p. 34-40, 2001.

PINCELLI, V. A.; BOCHIO, M. M.; MORIKAWA, M. K.; PEREIRA, P. M. Incidência e tratamento de cães com reações transfusionais agudas. Revista Clínica Veterinária, São Paulo, Ano XV, n. 86, p. 62-66, 2010.

PRACTICE guidelines for perioperative blood transfusion and adjuvant therapies. An updated report by the American Society of Anesthesiologists task force on perioperative blood transfusion and adjuvant therapies. Anesthesiology, Filadélfia, v. 105, n. 1, p. 198-208, 2006.

PRITTIE, J. E. Controversies related to red blood cell transfusion in critically ill patients. Journal of Veterinary Emergency and Critical Care, San Antonio, v. 20, n. 2, p. 167-176, 2010.

RABELO, R. C.; ARNOLD, C. F.; ALSUA, S. C. RICO Score - classificação rápida de sobrevida em cuidados intensivos. Variáveis inter-relacionadas em cães. Revista Clínica Veterinária, São Paulo, n. 78, p. 28-38, jan./fev. 2009.

RABELO, R. C.; CROWE JUNIOR, D. T. Fundamentos da terapia intensiva veterinária em pequenos animais condutas do paciente crítico. Rio de Janeiro: L.F. Livros, 2005 .

SAKR, Y.; LOBO, S.; KNUEPFER, S.; ESSER, E.; BAUER, M.; SETTMACHER, U.; BARZ, D.; REINHART, K. Anemia and blood transfusion in a surgical intensive care unit. Critical Care, Londres, v. 14 , p. 1-10, 2010 .

SCHWARTZ, S.; FRANTZ, R.; SHOEMAKER, W. Sequential hemodynamic and oxygen transport responses in hypovolemia, anemia and hypoxia. American Journal of Physiology, Bethesda, v. 241, n. 6, p. 864-872, 1981.

SHANDER, A. Anemia in the critically ill. Critical Care Clinics, Philadelphia, v. 20, n. 2, p. 159-178, 2004.

SIGRIST, N. E. Anemia and transfusion in critical illness. 
In: INTERNATIONAL VETERINARY EMERGENCY \& CRITICAL CARE SYMPOSIUM, 14., 2008, Phoenix. Proceedings... Phoenix: Omnipress, 2008, p. 781-783.

TILLEY, L. P. Essentials of canine and feline electrocardiograph. 3. ed. Malvern: Philadelphia London, 1992.

TOCCI, L. J. Transfusion medicine in small animal practice. Veterinary Clinics of North America: Small Animal Practice, Philadelphia, v. 40, n. 3, p. 485-494, 2010 .

TVEDTEN, H.; WEISS, J. D. Classification and laboratory evaluation of anemia. In: FELDMAN, B.F.; ZINKL, J. G.; JAIN, N. C. Schaml's veterinary hematology. 5. ed. Filadélfia: Lippincott Willians \& Wilkins, 2000. p. 143-150.

VINCENT, J. L.; YALAVATTI, G. Transfusion practice in the ICU: when to transfuse? Indian Journal of Critical
Care Medicine, Bombaim v. 7, n. 4, p. 237-241, 2003.

WANG, J. K.; KLEIN, H. G. Red blood cell transfusion in the treatment and management of anemia: the search for the elusive transfusion trigger. Vox Sanguinis, Basileia, v. 98, n. 1, p. 2-11, 2010.

WEISKOPF, R. B.; FEINER, J.; HOPF, H. W.; VIELE, M. K.; WATSON, J.; LIEBERMAN, J.; KELLEY, S.; TOY, P. Heart rate increases linearly in response to acute isovolemic anemia. Transfusion Medicine, Oxford, v. 43, n. 2, p. 235-240. 2003.

WEISS, D. J.; WARDROP, K. J. Schalm's veterinary hematology. 6. ed. Ames: Wiley-Blackwell, 2010. 1232 p.

WILKERSON, D. K.; ROSEN, A. L.; SEHGAL, L. R.; GOULD, S. A.; SEHGAL, H. L.; MOSS, G. S. Limits of cardiac compensation in anemic baboons. Surgery, Chicago, v. 103. n. 6, p. 665-670, 1988. 